\title{
Determination of the molecular weight of a novel hydroxy-terminated cyclohexene oxide and epichlorohydrin copolyether
}

\author{
Li Hua, Ying-hua Qiao, Shan-shan Jin and Ya-dong Zhang \\ School of Chemical and Energy Engineering, Zhengzhou University, Zhengzhou, 450001, China.
}

Accepted 11 January, 2018

\begin{abstract}
Cyclohexene oxide $(\mathrm{CHO})$-epichlorohydrin $(\mathrm{ECH})$ copolyether is one of the raw materials to synthesize transparent polyurethane. In order to control the performance of product, it requires the determination of molecular weight. But at present, the molecular weight of $\mathrm{CHO}-\mathrm{ECH}$ copolyether has not been reported. Therefore, in this paper, the molecular weight and constant $\mathrm{K}$ and $\alpha$ of the solution system was determinated by viscosity method based on Mark-Houwink empirical equation and intrinsic viscosity. This method provides a theoretical basis for the determination of molecular weight of polyether polyols.
\end{abstract}

Keywords: Copolyether, viscosity method, Ubbelohde viscometer, Mark-Houwink empirical equation.

*Corresponding author. E-mail: lihua@zzu.edu.cn. Fax: 0086-371-63886154.

\section{INTRODUCTION}

Polyurethane synthesized by $\mathrm{CHO}-\mathrm{ECH}$ copolyether has good transparency, which the six-member ring can improve the wear resistance and heat resistance of the polymer, and the introduction of chlorine atom will makes polyurethane has some new features, such as flame resistance, good adhesion and so on (Liu et al., 2005). The relative molecular mass of polymer reflects its size, and relates to its physical properties (Wang and Lin, 2004). The molecular weight of $\mathrm{CHO}-\mathrm{ECH}$ copolyether has an important influence on the control of the performance of the product in the processing and manufacturing. Therefore, it is great significance to find a simple, convenient and fast method to measure the polymer molecular weight. There are many methods to measure the relative molecular mass of polymer, such as acid value method, it was simple and easy, but interference factors is so many, the reagent is harmful, the error range is larger than $20 \%$. Since Mark-Houwink established the empirical relationship $[\eta]=\mathrm{KMn}^{\alpha}$ between the intrinsic viscosity [ $\eta$ ] of polymer solution and average molecular mass Mn of polymer, it is an effective way to measure the molecular mass with viscosity method to control the quality of the polymer. Viscosity method has the advantages of simple equipment, convenient operation, short processing cycle, high precision and so on. At present, there is no report on the study of the molecular mass of $\mathrm{CHO}-\mathrm{ECH}$ polymer so far, which is mainly due to the lack of the characteristic parameters $\mathrm{K}$ and $\alpha$ (characteristic constant) of the solution system. K value is related to the solution properties and the average molecular weight of solute, a mainly depends on the morphology of the polymer in solution (Liu and Fu, 2011).

In this paper, the molecular weight and constant $\mathrm{K}$ and $\alpha$ of the solution were measured by viscosity method based on Mark-Houwink equation. The method provides a theoretical basis for the measurement of molecular mass of polyether polyols.

\section{EXPERIMENTAL}

\section{Instruments and reagents}

$\mathrm{N}, \mathrm{N}$ dimethyl formamide, dichloromethane, cyclohexene oxide, epichlorohydrin, anhydrous alcohol, were of AR grade and obtained from Shanghai Chemical Reagent Co. 
and have mass fraction purities of 0.995 .0 .5 to $0.6 \mathrm{~mm}$ Ubbelohde viscometer, thermostat water bath, electronic balance, stopwatch and microthermometer.

\section{Experimental process}

\section{Synthesis of polyether polyols}

Cyclohexene oxide and epichlorohydrin were mixed and dripped into dichloromethane solution containing catalyst, then reacted for some time at $0^{\circ} \mathrm{C}$. After the reaction, washing the crude product, distillation at the low pressure then pure polyether polyol was obtained (Liu et al., 2005).

\section{Measurement of molecular weight by titration}

This is carried out by taking about $3 \mathrm{~g}$ polyether, adding $25 \mathrm{ml}$ acylation reagent, heating $25 \mathrm{~min}$ at about $98^{\circ} \mathrm{C}$ water bath, then cooling to room temperature, adding 20 $\mathrm{ml}$ hydrolyzate to the coolant liquid and dropping of phenolphthalein, titration with potassium hydroxide ethanol solution (Zhou and Deng, 2012). Average molecular weight $\mathrm{Mn}$ of polymer can be obtained by Equations 1 and 2:

$$
\mathrm{X}=\left(\mathrm{V}_{0}-\mathrm{V}_{1}\right) \cdot \frac{\mathrm{C}}{\mathrm{m}}
$$

$X$ : hydroxyl value, $\mathrm{mmol} / \mathrm{g}$;

$V_{1}: \mathrm{KOH}$ standard solution volume for test sample, $\mathrm{ml}$.

$V_{0}: \mathrm{KOH}$ standard solution volume for blank test sample, $\mathrm{ml}$.

$C: \mathrm{KOH}$ concentration of standard solution, $\mathrm{mol} / \mathrm{L}$.

$m$ : mass of the sample, $\mathrm{g}$.

$$
\mathrm{Mn}=\frac{\mathrm{n} \times 1000}{\mathrm{X}}
$$

$\mathrm{Mn}$ : Average molecular weight of copolyether, $\mathrm{g} / \mathrm{mol}$. $n$ : number of analyzed groups.

\section{Measurement of viscosity}

Viscosity was measured using an Ubbelohde-type glass capillary viscometer of 0.5 to $0.6 \mathrm{~mm}$ diameter (Shanghai Glass Instruments Factory, China), calibrated with double-distilled water at 298.15 and $313.15 \mathrm{~K}$. A thoroughly cleaned and dried viscometer, filled with experimental solutions, was placed vertically in an insulated jacket, wherein constant temperature $( \pm 0.02 \mathrm{~K})$ was maintained by circulating water from a thermoelectric controller (type 501, Shanghai Laboratory Instrument Works Co., Ltd.) at the required temperature. After thermal stability was attained, the flow times of the solutions were recorded with an electronic digital stopwatch correct to $\pm 0.01 \mathrm{~s}$. At least five repetitions of each datum point obtained were reproducible to $\pm 0.06 \mathrm{~s}$, and the results were averaged. Because all flow times were greater than $200 \mathrm{~s}$ and the capillary diameter ( 0.5 to $0.6 \mathrm{~mm}$ ) was far less than its length $(120 \mathrm{~mm})$, the kinetic energy and end corrections, respectively, were found to be negligible. DMF was as the solvent, outflow time was as $t_{0}$, the viscosity was then calculated from the fundamental relationship.

\section{Measurement of average molecular weight Mn of polymer}

Mark-Houwink empirical equation can be expressed as Ni (2012):

$[\eta]=\mathrm{KMn}^{\alpha}$

In which, $[\eta]$ is the intrinsic viscosity of polymer solution $M n$ is average molecular weight of polymer,

$K, \quad \alpha$ : the constants at a given temperature and characteristic of the solvent and the solute.

Therefore as long as [ $\eta]$ was gotten, $M n$ could be obtained by the formula.

\section{RESULTS AND DISCUSSION}

\section{Calculation of intrinsic viscosity [n]}

$\eta_{1}$ represents viscosity of solution, $\eta_{0}$ represents viscosity of the pure solvent.

Relative viscosity $\eta_{r}: \eta_{\mathrm{r}}=\frac{\eta_{1}}{\eta_{0}}$

Specific viscosity $\eta_{s p}: \eta_{\mathrm{sp}}=\frac{\eta_{1}-\eta_{0}}{\eta_{0}}=\eta_{\mathrm{r}}-1$

Based on Poiseuille formula (Guo et al., 2009): $\eta=$ $\frac{\pi r^{4} \text { thg }}{8 \operatorname{lv}}$

Where, $v$ : volume of liquid flowing through capillary; $r$ : capillary radius; $\rho$ : density of liquid; $l$ : the length of the capillary; $t$ : time of outflow; $h$ : average liquid column height; $g$ : acceleration of gravity.

For the same viscometer, $h, r, l, v$ is constant, then $\eta=$ K'pt. (7)

Suppose, $t_{0}$ and $t_{1}$ were the outflow time of pure solvent and solution under the condition of constant temperature, $\rho_{0}$ and $\rho_{1}$ were the density of pure solvent and solution, respectively. Under normal conditions, measurement of viscosity was carried out in dilute solution, the density of 
solution $\rho_{1}$ and the density of pure solvent $\rho_{0}$ can be regarded as equal $(\mathrm{GB} / \mathrm{T} 1632.1-2008,2008)$. Then the relative viscosity $\eta_{r}$ of the solution can be expressed as:

$\eta_{\mathrm{r}}=\frac{\eta_{1}}{\eta_{0}}=\frac{\mathrm{K}^{\prime} \rho_{1} \mathrm{t}_{1}}{\mathrm{~K}^{\prime} \rho_{0} \mathrm{t}_{0}} \approx \frac{\mathrm{t}_{1}}{\mathrm{t}_{0}}$

It can be seen that as long as the outflow time $t_{1}$ of the polymer solution at different concentration and outflow time $t_{0}$ of pure solvent were measured, then $\eta_{r}$ and $\eta_{s p}$ at different concentration can be calculated. According to the empirical formula (Xiang and Yu, 2009):

$\frac{\eta_{\mathrm{sp}}}{\mathrm{c}}=[\eta]+\mathrm{k}[\eta]^{2} \mathrm{c}$

$\frac{\ln \eta_{\mathrm{r}}}{\mathrm{c}}=[\eta]-\beta[\eta]^{2} \mathrm{c}$

Plotting the curve of $\frac{\eta_{\mathrm{sp}}}{\mathrm{c}}-\mathrm{c}$ and $\frac{\ln \eta_{\mathrm{r}}}{\mathrm{c}}-\mathrm{c}$, and finding intersections on the two lines, that is the intrinsic viscosity $[\eta]$. The relationship between viscosity and concentration is shown in Figure 1.

\section{Determination of $K$ and $\alpha$}

Average molecular weight can be calculated by using Equation 3.

Because the solvent-solute in this paper is a new system, values of $K$ and $\alpha$ were unknown, we take logarithm of both sides for above empirical equation, then it becomes:

$$
\ln [\eta]=\ln \mathrm{K}+\alpha \ln \mathrm{Mn}
$$

If the curve of $\ln [\eta]-\operatorname{lnMn}$ was plotted, $\alpha$ is the slope of the straight and InK is the intercept, thus average molecular weight can be calculated by using Equation 3 as shown in Table 1.

The curve of $\operatorname{In}[\eta]-I n M n$ was shown in Figure 2. From Figure 2, it can be obtained:

$\ln [\eta]=0.5362 \ln \mathrm{Mn}-2.7358$

Therefore $K$ and $\alpha$ can also be calculated: $K=0.06484, \alpha$ $=0.5362$, and then, the average molecular weight of polymer can be obtained.

\section{Measurement of molecular weight and test}

The molecular weight of the above samples was determined by viscosimetry and was compared with chemical method; the results were shown in Table 2.

The viscosimetry was proved to be feasible comparing with chemical method to detect the polymerization degree of $\mathrm{CHO}-\mathrm{ECH}$ copolyether.

Average molecular weight determined by viscosity method has higher linearity and precision in the measuring range of molecular weight of $10^{3}$ to $10^{7}$. If the concentration is too low, the error of outflow time will be greatly increased, and a very slight change in $t_{1}$ and $t_{0}$ brought the larger error of $\eta_{r}$; On the contrary, if the concentration is too high, the solute occurs serious agglomeration, and their relation will not be linear, the

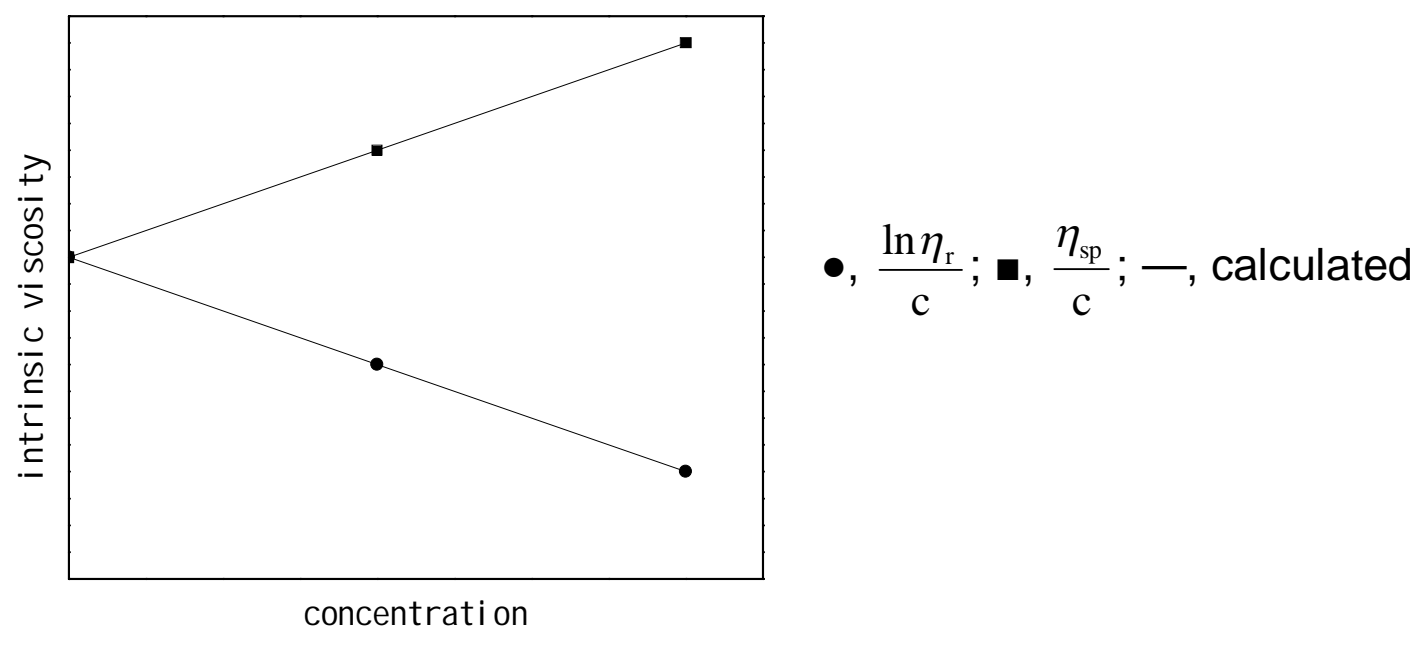

Figure 1. The relationship between intrinsic viscosity and concentration. 
Table 1. The result determined by viscosity method.

\begin{tabular}{lccccc}
\hline Parameter & $\mathbf{1}$ & $\mathbf{2}$ & $\mathbf{3}$ & $\mathbf{4}$ & $\mathbf{5}$ \\
\hline Intrinsic viscosity, N/mm & 2.996 & 3.5243 & 3.7057 & 3.9672 & 4.4324 \\
Average molecular weight & 1221.150 & 1856.630 & 2022.245 & 2091.380 & 2447.620 \\
$\ln [\eta]$ & 1.0985 & 1.2597 & 1.3099 & 1.3780 & 1.4889 \\
$\operatorname{lnMn}$ & 7.1075 & 7.5265 & 7.6120 & 7.6484 & 7.8029 \\
\hline
\end{tabular}

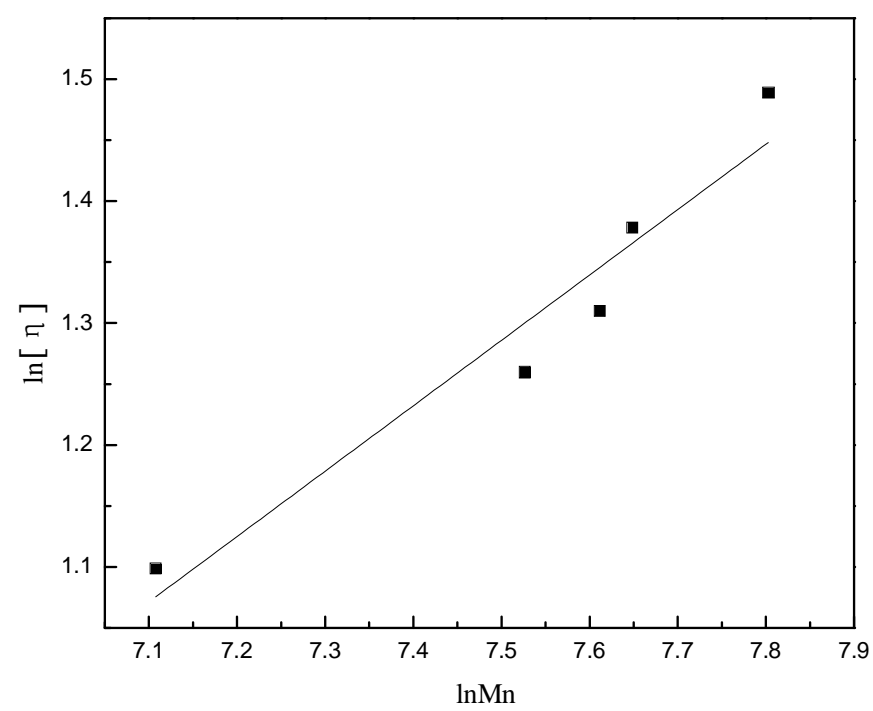

Figure 2. The relationship between $\ln [\eta]$ and $\ln \mathrm{Mn}$.

Table 2. Comparison of molecular weight determined by chemical method and viscosimetry.

\begin{tabular}{lccccc}
\hline Molecular weight & $\mathbf{1}$ & $\mathbf{2}$ & $\mathbf{3}$ & $\mathbf{4}$ & $\mathbf{5}$ \\
\hline Viscosimetry & 1266.37 & 1718.51 & 1889.89 & 2150.42 & 2652.93 \\
Chemical method & 1221.15 & 1856.63 & 2022.245 & 2091.38 & 2447.62 \\
\hline
\end{tabular}

results of extrapolation are not reliable. Therefore, it is necessary to choose the appropriate concentration, which makes $\eta_{r}$ between 1.2 and 2.0 (Guo et al., 2014).

\section{CONCLUSION}

Measurement of molecular weight of polymers has important significance for controlling the performance of product. In this paper, the molecular weight of polymers was measured by using viscosity method, the characteristic parameters $K$ and $\alpha$ of the solution system were obtained through the Mark-Houwink equation. The results of viscosity method showed a good agreement with the chemical method. The viscosity method is simple, convenient, low cost, high precision, so it has some advantages in the measurement of molecular weight of polymer. This method provides a theoretical basis for the determination of molecular weight of polyether polyols.

\section{REFERENCES}

GB/T1632.1-2008, (2008). Plastics-determination of the viscosity of polymers in dilute solution using capillary viscometers.

Guo F., Li C. F., Wei J. H. (2009). Discussion on experimental process of determining viscosity average molecular weight of UHMWPE by viscosity method. Plastics Sci Technol, 37(9): 62-66.

Guo X.P., Kang X. J., Li J. Y. (2014). Theory preparatory discussion about viscosity determination method for molecular weight of PPS. China Fiber Inspection, 33(21): 74-76.

Liu D. F., Fu Y. (2011). Determination of molecular weight and K, a values for polymers of glycidyl methacrylate. 29(13): 62-65.

Liu Y. C., Deng J. R., Ma J. Y. (2005). Synthesis and characterization of hydroxyterminated CHO-ECH copolyether. Polym Mater Sci Eng, 21(6): 73-76.

Ni, S. L. (2012). Testing and characterization of polymer molecular weight. Guangdong Chemical Industry, 39(2): 190-193.

Wang Y. Z., Lin Y. L. (2004). Studies of reasons of success or failure in experiment of viscosity measurements to calculate relative molecular weight of high polymer. J Jianghan Univ (Nat Sci), 32(4): 58-60. 
Xiang S. L., Yu R. T. (2009). Improvement of the experiment for measuring the molecular weight of polymer by measuring its intrinsic viscosity. Exper Sci Technol, 7(5): 37-39.

Zhou J., Deng J. R. (2012). The study of synthesis and characterization of the hydroxyl-terminated cyclohexene oxide-tetrahydrofuran copolyether.
Citation: Hua L., Qiao Y., Jin S., Zhang Y. (2018). Determination of the molecular weight of a novel hydroxy-terminated cyclohexene oxide and epichlorohydrin copolyether. Afr J Eng Res, 6(1): 10-14. 